\title{
Managing Patient Flows in Radiation Oncology - Reworking Existing Treatment Designs to Prevent Infections During the COVID-19 Pandemic at a German University Hospital
}

\section{Dennis Akuamoa-Boateng, MBA ( $D$ Dennis.Akuamoa-Boateng@uk-koeln.de)}

Department of Radiation Oncology, Center for Integrated Oncology, University Hospital Cologne, Cologne, Germany https://orcid.org/0000-0002-9355-8470

\section{Simone Wegen, MD}

Department of Radiation Oncology, Center for Integrated Oncology, University Hospital Cologne, Cologne, Germany Justin Ferdinandus, MD

Department of Nuclear Medicine, University Hospital Essen, Essen, Germany

\section{Regina Marksteder}

Department of Hospital Pharmacy, University Hospital Cologne, Cologne, Germany

\section{Christian Baues, MD}

Department of Radiation Oncology, Center for Integrated Oncology, University Hospital Cologne, Cologne, Germany

\section{Simone Marnitz, MD}

Department of Radiation Oncology, Center for Integrated Oncology, University Hospital Cologne, Cologne, Germany

\section{Keywords:}

Posted Date: June 13th, 2020

DOI: https://doi.org/10.21203/rs.3.rs-34056/v1

License: (9) This work is licensed under a Creative Commons Attribution 4.0 International License. Read Full License 


\section{Abstract}

The authors have requested that this preprint be removed from Research Square. 